\title{
The Gödel incompleteness theorems (1931) by the axiom of choice
}

\author{
Vasil Penchev, vasildinev@gmail.com \\ Bulgarian Academy of Sciences: Institute of philosophy and Sociology: \\ Dept. of Logic and Philosophy of Science
}

\begin{abstract}
Those incompleteness theorems mean the relation of (Peano) arithmetic and (ZFC) set theory, or philosophically, the relation of arithmetical finiteness and actual infinity. The same is managed in the framework of set theory by the axiom of choice (respectively, by the equivalent well-ordering "theorem'). One may discuss that incompleteness form the viewpoint of set theory by the axiom of choice rather than the usual viewpoint meant in the proof of theorems. The logical corollaries from that "nonstandard" viewpoint the relation of set theory and arithmetic are demonstrated.
\end{abstract}

Key words: choice, arithmetic, set theory, well-ordering, information

Many efforts address the constructiveness of the proof in the so-called Gödel first incompleteness theorem (Gödel, 1931). One should use the axiom of choice instead of this and abstract from that the theorem is formulated about statements, which will be replaced by the arbitrary elements of any infinite countable set. Furthermore all four theorems ( Gödel, 1931; Löb, 1955; Rogers, 1967; Jeroslow, 1971) about the so-called metamathematical fixed points induced by the Gödel incompleteness theorems will be considered jointly and in a generalized way.

The strategy is the following:

An arbitrary infinite countable set "A" and another set "B" so that their intersection is empty are given. One constitutes their union " $\mathrm{C}=\mathrm{A} \cup \mathrm{B}$ ", which will be an infinite set whatever " $\mathrm{B}$ " is. Utilizing the axiom of choice, a one-to-one mapping " $f=A \leftrightarrow C$ " exists. One designates the image of "B" into "A" through "f" by " $B_{f}$ " so that " $B_{f} \subset \mathrm{A}$ ". If the axiom of choice holds, there always an internal and equivalent image like " $B_{f}$ " for any external set like "B". Thus, if one accepts that " $B_{f} \equiv \mathrm{B}$ ", whether an element "b" of " $\mathrm{B}$ " belongs or not to " $\mathrm{A}$ " is an undecidable problem as " $b_{f} \equiv \mathrm{b} "$.

However if the axiom of choice is not valid, one cannot guarantee that "f " exists and should display how a constructive analog of "f " can be built. If one shows how "f " to be constructed at least in one case, this will be a constructive proof of undecidability as what Gödel's is.

Another option is to prove that no constructive analog exists for any mapping "f" guaranteed by the axiom of choice. In a sense, this would be an analog of the theorems about the absence of hidden variables in quantum mechanics (Neumann, 1932; Kochen, Specker, 1968). John von Neumann initially and Simon Kochen and Ernst Specker afterwards also managed to paraphrase the internal consistency of infinity corresponding to the reformulation of Henkin's proposition in the same terms. Neumann deduced it from the availability of noncommutative operators in the mathematical formalism of quantum mechanics founded on Hilbert space while Kochen and Specker did the same on the ground of wave-particle dualism even if the corresponding operators are commutative.

Furthermore the corollaries of these theorems can demonstrate the intimate and extraordinary link between quantum mechanics and the axiom of choice: one link inherited in fact yet from the infinity admitted in quantum mechanics: 
According to them, any well-ordering in any quantum system is excluded before measurement being a coherent and inseparable whole. Nevertheless it represents a well-ordered statistical ensemble of results after measurement. The basic epistemological postulate requires the states before and after measurement to be equated and thus the well-ordering theorem equivalent to the axiom of choice to be utilized necessarily to equate an unorderable set (before measure-ment) with a wellordered set (after measurement).

However the coherent state before measurement excludes the axiom of choice just as these theorems are valid. Thus the mathematical structure used by quan-tum mechanics should be invariant to the axiom of choice in a sense. Indeed Hilbert space together with its identical dual space can describe equally success-fully a wellordered infinitely-dimensional vector and the unorderable character-istic function of the probabilistic distribution of the values of its components.

Thus quantum mechanics interpreted as a theory about infinity can transfer rather instructive conclusions into set theory concerning a possible and undeveloped yet probabilistic interpretation of infinity and therefore, that of the set theory itself. All this allows the further discussed entanglement, quantum information, and quantum computer to be interpreted in terms of set theory and seen from the viewpoint of the generalization of Henkin's proposition.

Coring the reformulation, the problem of (in)completeness can be general-ized as a property of all infinite sets. An infinite set unlike any finite one can be both complete (universal) and incomplete (open) in a sense reminiscent to the "clopen" (both closed and open) sets in topology such as all discrete sets are. There is a hidden, but intimately link between discreteness and infinity. Quantum mechanics, forced to introduce quanta and thus discreteness, has therefore introduced infinity in an experimental and exact science such as physics.

What is that bridge is what leads from Henkin's proposition to quantum computer, both being from the "internal side" of completeness. The internal position to infinity as to a quantum computer means the following: Any potential "user" should be sited inside the quantum computer before the beginning of the computation so that user's observation on the quantum computer is in-cluded in advance in the result which will be obtained ultimately. Under that condition, the quantum computer can resolve any problem, and it cannot "hang up" ever. As any quantum computation is equivalent to a wave function of the universe in general and thus to one state of it, mankind observes constantly this property of a quantum computer: Indeed the universe, to which mankind is sited always inside, never "hangs up" and any physical process finishes in a finite time. A quantum computer to an internal observer knows its state and resolves the "halting problem" as its computation ends always with a result. David Albert $(1983,1987)$ proved the same in another way coining the term "quantum automaton" for a quantum computer.

What about a quantum computer locked in a "Chinese room" therefore forcing an external position to it? First of all, a quantum "Chinese room" should admit an arbitrary degree of entanglement between the quantum computer inside and the interviewers outside, a kind of "quantum telepathy". Then the axiom of choice will be partially valid to the extent exactly defined by that degree of entanglement. Consequently the quantum computer in any degree of entanglement cannot accomplish the reordering right, to the utterance and it will hang up in general. However in fact the quantum computer locked in the "Chinese room" will be replaced by another quantum computer including both the interviewers outside the "Chinese room" and the former inside it. The latter quantum computer will give the answer for the former always as it includes the "users" of it while the interviewers will think that the answer is given by the locked one, which will "hang up" and thus mute.

What turns out to be the case? As if a quantum computer cannot be locked in

a "Chinese room" as a law of nature. However it will fail in the "Chinese room 
test" in a paradoxical way: It will give the right answer always even ostensibly "locked" while a human being can be really deprived of the context outside the "Chinese room" and thus give a different answer in general.

The "Chinese room" allows of a curious visualization of the invariance to the axiom of choice as to a quantum computer: The invariance means that the hung-up quantum computer inside and the entire quantum computer including that inside together with the interviewers can be discussed as equivalent as quantum systems described in Hilbert space(s). The same can be formulated also so: The quantum computer is only a single one, and all including mankind is within it necessarily.

Henkin's proposition is the "formula" defined in "a problem concerning provability" (Henkin, 1952). It is commonly interpreted as the opposite to the Gödel proposition stating the proper unprovability under the same conditions.

The statements can be also reformulated in two ways, which are not selfreferential:

"Jeroslow's proposition": a formula stating the provability of another under the same conditions (Jeroslow, 1971).

"Rogers' proposition": a formula stating the unprovability of another under the same conditions (Rogers, 1967).

The reformulation of all four cases in the present context is the following:

An element "a" of an infinite countable set "A" and an element "b" of another set "B" are given, and " $b_{f}$ " designates the image of "b" after " $A \cup \mathrm{B}$ " is counted by "A" [" $\mathrm{f}=(A \cup B) \leftrightarrow A$ "]:

H: The reformulation of Henkin's (Löb's) case: " $\left(\mathrm{a} \equiv b_{f}\right) \wedge(\mathrm{B} \subset \mathrm{A})$ "

G: The reformulation of Gödel's case: " $\left(\mathrm{a} \equiv b_{f}\right) \wedge(\mathrm{B} \cap \mathrm{A}=\emptyset)$ "

J: The reformulation of Jeroslow's case: " $\left(\mathrm{a} \not \equiv b_{f}\right) \wedge(\mathrm{B} \subset \mathrm{A})$ "

R: The reformulation of Roger's case: " $\left(\mathrm{a} \not \equiv b_{f}\right)(\mathrm{B} \cap \mathrm{A}=\emptyset)$ "

The reformulations are more general for they abandon:

$\rightarrow$ The specification of the elements both of "A" and "B" as any propositions;

$\rightarrow$ The specification of " $f$ " as any constructive encoding of the elements of " $A \cup \mathrm{B}$ " including the Gödel numbering.

However it needs the axiom of choice to guarantee the existence of " $f$ " in any case.

Consequently, the proof of the reformulation of Henkin's case would be a proof of the ZFC consistency ("ZFC" means the set theory founded on the axioms of Zermelo, Fraenkel plus the axiom of choice). Indeed the strategy of ZFC involves as "sets" those entities ("classes"), which are true subsets or parts of another entity ("class"). Then one can say about any proposition in ZFC that it has an image as an element of some set definable in ZFC and thus the ZFC set theory represents its proper consistency just as Henkin's proposition states its provability.

Even more, any other axiomatics of set theory should be necessarily equivalent to it as Gödel's, Jeroslow's, and Rogers' propositions are undecidable and one can conjecture the same in a sense about their reformulations as above.

Martin Löb (1955) proved that Henkin's proposition (1952) is provable:

"If $S$ is any formula such that $\tilde{B}(S) \rightarrow S$ is a theorem, then $S$ is a theorem" 
The corollary is: "The particular formula $S$ of Henkin's problem, which is the same as $\tilde{B}(S)$, is a theorem."

One can reformulate in a trivial way his proof so that it holds in the reformulation of Henkin's case as above. Indeed almost all proof of Martin Löb concerns the Gödel encoding to be validated under the conditions of the theorem. If one accepts the above generalizing reformulation, its statement seems to be obvious:

$" b_{f} \rightarrow b "\left(\right.$ as $\left." b_{f} \equiv b "\right)$ implies " $b \in \mathrm{A} "$ (as " $\left.b \in \mathrm{B} \subset \mathrm{A} "\right)$. This is not true in the rest three cases (the reformulations Gödel's, Jeroslow's, and Rogers' proposition).

The only positive result (about Henkin's propostion) as well as the rest three, which can be considered as negative, can be interpreted also thus: The only consistent position to infinity is the internal one. As set theory involves infinity, its axiomatics such as ZFC should guarantee just the internal position for itself to be consistent.

\section{References}

Albert, D. (1983). On quantum-mechanical automata. Phys. Lett. A, 98, 249252.

Albert,D. (1987). A Quantum-Mechanical Automation. Philos. Sci., 54, 577585.

Gödel, K.(1931). Über formal unentscheidbare Sätze Principia mathematica und verwandter Systeme I. Monatsh. Math. Phys., 38 , 173-198.

Henkin, L. (1952). A problem concerning provability, problem 3. J. Symb. Logic, 17,160 .

Jeroslow, R. (1971). Consistency statements in formal mathematics. Fund. Math., 72 , 17-40.

Kochen,S., Specker, E. (1968). The problem of hidden variables in quantum mechanics. J. Math. Mech., 17,, 59-87.

Löb, M. (1955). Solution of a problem of Leon Henkin. J. Symb. Logic, 20, $115-118$.

Neumann, J. von (1932) Mathematische Grundlagen der Quantenmechanik. Berlin: Springer, 1932.

Rogers, H. Theory of Recursive Functions and Effective Computability. New York: McGraw Hill, 1967. 